\title{
A Mathematical Modelling with Zakharov System for Langmuir Wave in Unmagnetized Plasma
}

\author{
Sudarshan Kumar Chakravarti* \\ University Department of Physics, T. M. B. University, Bhagalpur, India \\ *Corresponding author: sudarshanchakravarti5@gmail.com
}

\begin{abstract}
In this article we present a discussion and overview of mathematical result of the self-focusing of a Langmuir wave which governs Zakharov system and has studied the self- focusing of a Langmuir wave following by Gaussian distribution. Langmuir wave propagates through uncharged plasma which governed by Zakharov systems. The phenomenon plays a vital role in the Dynamics. We present the article mathematical model with effect of Landou damping. Relativistic mass oscillation and ponderomotive force on electrons of the ionized plasma encouraged the Langmuir wave which resists the self-focusing effect when damping is ignored. The Beam radius gets narrow. when it further propagates considering the paraxial ray's approximation, the self-focusing length $R_{n}$. It shows that characteristics of varying bandwidth distance of propagation in relativistic plasma.
\end{abstract}

Keywords: Langmuir wave, Landou damping, Self-focusing, Zakharov system.

\section{Introduction}

Laboratory and many space observations have proved that there have been lots of evidences for the existence of Langmuir wave [1]. Over last some years the propagation of Langmuir wave promotes a very facinate area of the research due to their huge applications in different plasma interactions [2]. Langmuir wave propagates in neutral plasma and governed by Zakharov system. It is a system of nonlinear partial differential equation [3]. At first Vlodimir Zakharov in 1972 discussed the propagation of Langmuir wave in an ionised plasma [4]. Langmuir wave propagates in a unmagnetized or less magnetic plasma can be described with the help of Zakharov systems. In 1920 Langmuir wave discovered by Irvin Langmuir and Levi Tonks [5]. when plasma wave accelerate electron and its acceleration is in the comparision of phase velocity of the wave and this process performs in the form of damp mode which is known as Landau damping [6] when in any conducting media electron density changes its value fast oscillatory form in the ultraviolet region [7].

Collective charged particle accelerator has been centre of both theoretical and experimental research from last few years [8]. In this process two laser beam of different frequencies $\omega_{1}$ $\& \omega_{2}$ and wave number $K_{1}$ and $K_{2}$ respectively are established in plasma. They Express a ponderomotive force on the electrons and it resonantly a Langmuir wave of the the $\omega_{1}-\omega_{2} \approx \omega_{\mathrm{P}}$ and the wave number $\mathrm{k}_{1}-\mathrm{k}_{2} \approx \frac{\omega \mathrm{P}}{c}$. when plasma wave starts to propagates, the relativistic effects on the frequency discrepancy becomes useful in the plasma wave. It reaches saturation at the potential which is larger in present ponderomotive potential $\phi_{\mathrm{p}}$ $\left(\phi>>\phi_{\mathrm{p}}\right)$. The electron governs by Langmuir wave gets very large velocity and it can be accelerated to several $\mathrm{MeV}$ energy [9].

In this method, any dissimilarity in the intensity partition of the Langmuir waves can have important response on the particle oscillation process. So conscientious study of the propagation of dissimilar Langmuir wave is to be performed.

In the present paper we have studied the mathematical overview of the self-focusing of Langmuir wave generated by Zakharov system with a gaussian intensity distribution along wavefront. when laser beam propagates through the plasma it contributes the self-focusing, however, it may be ignored as long as oscillatory electron velocity overcomes following Langmuir wave, due to the laser beam. its shows $\phi>\left(\omega_{p}\right)$ $\left.\omega_{\text {laser }}\right) E_{\text {laser }} / k$, here $E_{\text {laser }}$ is the field of the laser beam. $\omega_{\text {laser }}$ and $\mathrm{k}$ iare the frequency and wave number of the laser beam respectively. Latest computer simulations have proved that the Langmuir wave is also responsible for self-focusing [10].

Although the present discussion is unpredictable to some particular extent because of refractive index modification affect. By Langmuir wave impact the propagation and intensity distribution of the laser beams. the propagation of Langmuir wave in homogeneous plasma can outcomes as self-focusing by developing a density depression in the ionized plasma and similarly by increasing the electron mass through relativistic effect [11].

Transverse ponderomotive force is mainly responsible for density depression, which repels plasma from high field reason to Low field region [12]. this density depression develops a perceptible increase in the refractive index of the refraction and pretends like optical guide for radiation beam [13]. Again this self-focusing mechanism, proceeded to reduce the plasma 
frequency growing in regions of high field intensity because of relativistic mass increment of the electrons under the Langmuir wave. Again the self-focusing because of density depression creates on a longer time scale which is responsible for increasing the relativistic mass in self-focusing effect [14].

Present applied process also ignores an important effect, for example the parametric coupling of Langmuir mode to the ionized acaustic wave. This opposes the analysis to $v_{\text {osc }}<$ $v_{\text {oscth }}$ where represents the thershold value of varying velocity for parametric excitation. But in the case of eikonal theory of coupled plasma mode is comfortable to use in the place of eikonal theory for a single mode. In part 2 we discussed a simple overview and analysis of self-focusing using eikonal approach. we determined an expression for the beam width parameter and in part 3 study its characteristics with distance of propagation.

\section{Determination of Beam Width Parameter}

If we consider the propagation of Langmuir wave in homogeneous plasma and its equilibrium density is $n_{o}^{o}$ then for harmonic time dependence of the potential oscilatory velocity due to the langmuir wave is given by,

$$
v_{o s c} \simeq-\frac{e k}{m_{o} \Upsilon \omega} \phi(r)
$$

which is function of space. it is due to modified electron mass in relativistic case,

$$
\mathrm{m}=m_{o}\left(1-\frac{v_{o s c}^{2}}{c^{2}}\right)^{-1 / 2}
$$

Here $m_{o}$ is rest mass of the electron and due to ponderomotive force on electrons,

$$
\mathrm{F}_{\mathrm{P}}=-\mathrm{m} \frac{\mathrm{r}}{2 \nabla}\left(v_{o s C}^{2}\right)
$$

it is responsible for redistribution of electrons density on the time scale $\sim \frac{a_{o}}{c_{s}}$, here $a_{0}$ represents the radial extent of the Langmuir wave and $\mathrm{c}_{\mathrm{s}}$ represents the ion sound speed.

Dispersion relation for Langmuir wave is given by,

$$
\omega^{2}=\omega_{P}^{2}+k^{2} v_{t h}^{2}
$$

and in the form of $i \nabla$ is given by,

$$
\left(\nabla^{2}+\frac{\omega^{2}-\omega_{p}^{2}}{v_{t h}^{2}}\right) \phi=0
$$

where $\omega_{p o}=\left(\frac{4 n_{o} e^{o}}{\Upsilon_{o} m_{o}}\right)^{1 / 2}$ is the surrounding plasma frequency,

$$
\Upsilon(\mathrm{r}, \mathrm{z})=\left(1+\mathrm{a}^{2}(\mathrm{r}, \mathrm{z})\right)^{1 / 2}
$$

Represents relativistic mass factor it shows the intensity of normalised Langmuir wave. $\mathrm{n}_{\mathrm{o}}$ is time dependent electron density, $\mathrm{m}_{\mathrm{o}}$ rest mas and $\mathrm{e}$ is the electron charge. $v_{\text {th }}$ denotes the electron thermal speed.

$$
\mathrm{a}^{2}(\mathrm{r}, \mathrm{z}) \text { at } \mathrm{r}=0
$$

Radiation field is responsible for producing index of refraction profile on axis having maximum value. again taking Rapid phase variation of $\phi$ is $\mathrm{e}^{\mathrm{ikz}}$. In continuous mode the modified electron density can be represented as,

$$
\begin{aligned}
\mathrm{n}_{\mathrm{o}} & =n_{o}^{o} \exp \left(-\frac{v_{o s c}^{2}}{v_{t h}^{2}}\right) \\
& =n_{o}^{o}\left(1-\frac{v_{o s c}^{2}}{v_{t h}^{2}}\right)
\end{aligned}
$$

Putting equation (4) in equation (1), we find that,

$$
\begin{aligned}
& \nabla^{2} \phi=n_{o}^{o} \exp \frac{\omega^{2}}{v_{t h}}\left(\epsilon_{o}+\epsilon_{2} \phi \phi^{*}\right)=0 \\
& v_{\mathrm{th}}=\left(\frac{2 T e}{\Upsilon m}\right)^{1 / 2}, \epsilon_{o}=1-\frac{\omega_{p o}^{2}}{\omega^{2}}, \\
& \epsilon_{2}=\frac{e^{2} k^{2}}{m_{o}^{2} \Upsilon^{2} \omega^{2} v_{t h}^{2}} \text { and } \omega_{\mathrm{po}}=\left(4 \pi n_{o}^{o} e^{2} / \mathrm{m}_{\mathrm{o}}\right)^{1 / 2}
\end{aligned}
$$

$\phi$ and k can be expressed as,

$$
\Phi=\mathrm{A}(\mathrm{r}, \mathrm{z}) \mathrm{e}^{\mathrm{i}(\omega t-\mathrm{kt})} \mathrm{k}=\frac{\omega}{v_{t h} \epsilon_{o}^{1 / 2}}
$$

Using WKB approximation it may be represented as applying in eikonal as the complex amplitude represented by where we can write,

$$
-2 \mathrm{ki} \frac{\partial A}{\partial z}+\nabla^{2} \mathrm{~A}+\frac{\omega^{2}}{v_{t h}^{2} \epsilon_{2}} \mathrm{AA}^{*}=0
$$

Using eikonal $\mathrm{S}$ the complex amplitude A expressed as,

$\mathrm{A}=\mathrm{A}_{\mathrm{O}}(\mathrm{r}, \mathrm{z}) \exp [-\mathrm{ik} \mathrm{S}(\mathrm{r}, \mathrm{z})]$

Here $A_{O}$ and $S$ are function of $r$ and $z$,

$2 \frac{\partial S}{\partial z}+\left(\frac{\partial S}{\partial r}\right)^{2}=\frac{1}{k^{2}}\left(\frac{\partial^{2} A_{O}}{\partial r^{2}}+\frac{1}{r} \frac{\partial A_{o}}{\partial r}\right) \frac{1}{A}+\frac{\epsilon_{2}}{\epsilon_{O}} A_{O}^{2}$

$\frac{\partial A_{o}^{2}}{\partial z}+\left(\frac{\partial^{2} s}{\partial r^{2}}+\frac{1}{r} \frac{\partial s}{\partial r}\right) A_{o}^{2}+\frac{\partial A_{o}^{2}}{\partial r} \frac{\partial s}{\partial r}=0$

Considering $\mathrm{z}=0$,

$A_{o}^{2}=A_{o o}^{2} e^{-r^{2} / a^{2}}$

We can write $A_{o}^{2}$ and S paraxial ray approximation as,

$\mathrm{S}=\frac{r^{2}}{2 \beta(z, t)}+\Psi(\mathrm{z}, \mathrm{t})$ 


$$
\begin{aligned}
& A_{o}^{2}=\frac{A_{o O}^{2}}{f^{2}} e^{-r^{2} / a^{2} f^{2}} \approx \frac{A_{o O}^{2}}{f^{2}}\left(1-\frac{r^{2}}{a^{2} f^{2}}\right) \\
& \beta=\frac{1}{f} \frac{d f}{d z}
\end{aligned}
$$

In equation (12) parameter $\mathrm{f}$ is proportional axial intensity and also the width of the beam, known as beam width parameter, At $\mathrm{z}=0, \mathrm{f}=1$ and $\frac{d f}{d z}=0$, is true for initially plane wavefront. And hence

$$
\frac{\partial^{2} f}{\partial z^{2}}=\frac{\left(\frac{1}{k^{2} a^{4}}-\frac{\epsilon_{2} A_{o o}^{2}}{\epsilon_{O} a^{2}}\right)}{f^{3}}
$$

which gives,

$$
f^{2}=z^{2}\left(\frac{1}{R_{d}^{2}}-\frac{1}{R_{n}^{2}}\right)
$$

here, focusing length,

$$
\begin{aligned}
& \mathrm{R}_{\mathrm{n}}=\mathrm{a} \sqrt{\frac{\epsilon_{o}}{\epsilon_{\mathrm{Z}} A_{o o}^{2}}} \text { and diffraction length, } \\
& \mathrm{R}_{\mathrm{d}=\mathrm{a}} \mathrm{a}^{2} \mathrm{k}
\end{aligned}
$$

\section{Landau Damping Response}

If one integrate the landau of damping term for the Langmuir wave, from equation(5), $\epsilon_{o}$ over complex term $\epsilon_{o}+i \epsilon_{i}$, here

$$
\epsilon_{i}=2 \sqrt{\pi}\left(\frac{\omega_{p}}{k v_{t h}}\right)^{2} \exp \left\{-\frac{\omega^{2}}{\omega^{2}-\omega_{p}^{2}\left(1-\frac{v_{o s c}^{2}}{v_{t h}^{2}}\right)}\right\}
$$

Similarly, the intensity distribution of the Langmuir wave is represented as,

$$
A_{o}^{2}=\frac{A_{o o}^{2}}{f^{2} e^{-2 k_{i}{ }^{2}} e^{-\frac{r^{2}}{a^{2} f^{2}}}}
$$

Here $k_{i}=\frac{k \epsilon_{i}}{2 \epsilon_{o}}$ so, the equation leading beam width parameter can be represented as,

$$
\frac{d^{2} f}{d z^{2}}=\frac{1}{R_{d}^{2} f^{3}}-\frac{\epsilon_{2} A_{o o}^{2} \exp \left(-2 k_{i} z\right)}{\epsilon_{o} a^{2} f^{3}}
$$

Solving equation (12) with parameters, $\frac{v_{o s c}}{v_{t h}} \approx 0.1, \frac{k v_{t h 0}}{\omega_{p 0}}=0.5$, $\omega_{0}=10^{11} \mathrm{~Hz}, \mathrm{n}_{0}=10^{14} \mathrm{~cm}^{-3}, \mathrm{a}=200 \mu \mathrm{m}$ and $\mathrm{T}=10 \mathrm{eV}$. and plotting, we get the result,

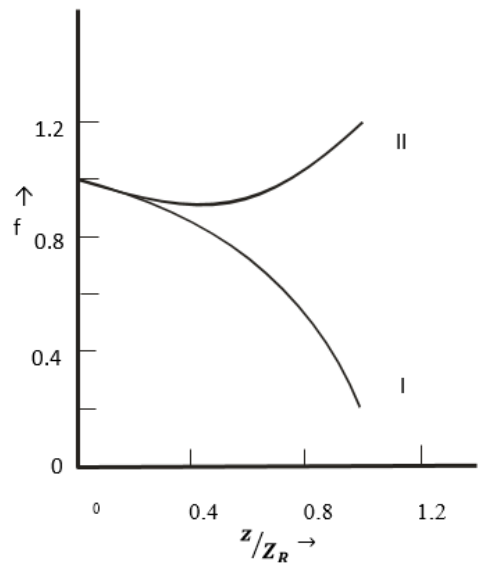

Fig. 1. Variation of the beamwidth parameter with the distance of propagation in a plasma. Curves I and II correspond to undamped and damped (kvth0 $\omega \mathrm{p} 0 \approx 0.25$ ) Langmuir waves

\section{Conclusion}

In this work, we find that the Langmuir wave of duration greater than $\mathrm{A} / \mathrm{C}$ and amplitude is taken such that is responsible for self-focusing in plasma. ponderomotive force is responsible for nonlinearity. But Landau damping of the Langmuir wave resists the self-focusing effect when damping is neglected. The Beam gets more focused through its propagation. But near the focal spot, when the intensity gets its peak and wave frequency is completely detuned form the local plasma density and WKB approximation not remains appropriate. The wave faces huge self-distortion; this is not desirable in the charged particle accelerator.

\section{References}

[1] Bethe, H.A. 1986, "Possible Explanation of the Solar-Neutrino Puzzle," Phys. Rev. Lett,56

[2] S.V. Nezlin, E.N and Trubnikov, "Modulation instability of Langmuir Wave exited in a plasma by an electron beam" Plasma Redaktsiiu, 25, 159-162.

[3] Cairans and A. Z., I.H 2000 "Generalized Langmuir Wave in Magnetized Plasma" Phy.Plasma, 7, 3167-3180.

[4] Shukla P.K. Eliasson B., "Colloquium: Nonlinear collective interactions in quantum plasmas with degenerate electron fluids," Rev. Mod.Phy, 83,885, 2011.

[5] P.H. Labelle, J. 2005 "Descreate Langmuir Wave in density Structure" Space Phy. J Geophy. Res110,11308

[6] Hass F. Elliason B. Shukla P. K., "Negative energy waves and quantum relativistic Buneman instabilities," Phys. Rev.E86.

[7] T. and Ito. T. Vlasov, "Simulation of Langmuir decay instability. Phy. Plasma, 15(8), 2008.

[8] W.L, Kruer, "The Physics of Plasma Interections", 1st edition, D. Pines, New York, 1988, P.104.

[9] R.L. Dewar, W, L, Kruer, and W. M Manheimer, "Modulational Instabilities Due to Trapped Electrons," Phys. Rev. Lett. 28, 215, 1972.

[10] B. D. Fried, H.W. Wild, J. Nuclear Energy, plasma Phys.1.190(1960)

[11] J, P. Holloway and J.J. Dorning, "Undamped longitudinal plasma waves," Phys. Lett. A 138, 279 (1989)

[12] R.J. 2004, Plasma wave and Electromagnetic Radiation at Venus and Mars," Adv. Space Res33,1956-1967.

[13] A.J. and Coirns, I. H. 2003, "Banded frequency structure from liner mode convertion in inhomogeneous plasma." Phy. Plasma, 10, 04072-4078.

[14] J. L. Kline, D. S, Montgomery, "Observation of a transition from kinetic to fluid nonlinearities for Langmuir Waves driven by stimulated Raman backscatter," Physics of Plasmas, vol. 12, 2005. 